13

\title{
Электроды вакуумной камеры под действием дуги в зоне взрывного плавления
}

\author{
(C) А.И. Кудюкин, М.Н. Махмудов, Е.Н. Моос, В.А. Степанов \\ Рязанский государственный университет им. С.А. Есенина, \\ 390000 Рязань, Россия \\ e-mail: a.kudykin@365.rsu.edu.ru
}

Поступило в Редакцию 11 декабря 2019 г.

В окончательной редакции 11 декабря 2019 г.

Принято к публикации 27 января 2020 г.

\begin{abstract}
Сплавные системы $\mathrm{Cu}(60 \%)-\mathrm{Cr}(40 \%)$ после многократного воздействия дугового вакуумного разряда обнаруживают изменение атомной концентрации меди (с 53.7 до 71.8 at.\%) и соответственно хрома. Массспектрометрия показала наличие в остаточной атмосфере диффузионных приборов фрагментов молекул типа $\mathrm{C}_{x} \mathrm{H}_{y}^{+}$и молекул, десорбируемых со всех деталей камеры, ионы остаточного газа $\left(\mathrm{CO}_{2}^{+}, \mathrm{H}_{2} \mathrm{O}^{+}, \mathrm{N}_{2}^{+}, \mathrm{N}^{+}\right.$ и их полиатомные композиции). Обнаружено наследование технологических примесей от предшествующих операций в масс-спектре.
\end{abstract}

Ключевые слова: вакуум, технологии, дугогасительная камера, масс-спектр, дуга, электроды, эрозия.

DOI: $10.21883 / J T F .2020 .07 .49458 .396-19$

\section{Введение}

Известны работы как по исследованиям вакуумной дуги, ее свойств в зависимости от токов и материала электродов, так и по эрозии поверхности различных сплавов под действием этой дуги [1-13]. При возникновении дуги в вакуумной дуговой камере (ВДК) контакты подвергаются сильному перегреву (до $3000^{\circ} \mathrm{C}$ ) [4], в особенности поверхностные слои, что провоцирует мгновенное (взрывное) расплавление и испарение металлов с поверхности контактов. Очевидно, что при температурах, в несколько раз превышающих температуры плавления материалов $\left(\mathrm{Cu}-1083^{\circ} \mathrm{C}, \mathrm{Cr}-\right.$ $1615^{\circ} \mathrm{C}$ ), на электродах следует ожидать необратимых изменений элементного состава, что, в свою очередь, должно сказаться на работоспособности самой дугогасительной камеры. В настоящей работе рассмотрены особенности перераспределения материалов электродных компонент и примесей в остаточной атмосфере ВДК, чтобы определить, что происходит со структурой электродов и составом окружающей газовой среды в условиях дугового вакуумного разряда.

\section{1. Методы исследования и образцы}

Для сплавных композиций вакуумных прерывателей неизвестно, в частности, как изменяется морфология поверхности контактов и состав в условиях интенсивных энергетических потоков. Последнее определило направленность настоящей работы, в которой указанная сплавная композиция изучалась методом атомносиловой микроскопии (АСM) и электронного микрозондового анализа (РСМА) совместно с растровым иссле- дованием морфологии поверхности образцов с помощью электронного микроскопа JEOL JSM-6610LV.

В литературе отсутствуют сведения по встраиванию квадрупольных систем масс-анализа в технологию производства вакуумных высоковольтных дугогасительных камер, что составило особенность исследований настоящей работы. Нами применялась квадрупольная система KMC-250 (разработка Рязанского предприятия „Шибболет“), которая зарегистрировала состав остаточных и рабочих газов на различных технологических этапах и после их завершения. В данной системе регистрации применен метод ионизации электронным ударом (при различных ускоряющих электроны напряжениях ионизатора).

При анализе ВДК, имеющей дефекты (некачественная пайка, поры или трещины в оболочке и т.п.), масс-спектрометрометр фиксировал изменение интенсивности гелия (при проверке герметичности оболочки) за счет его проникновения внутрь камеры (рис. 1).

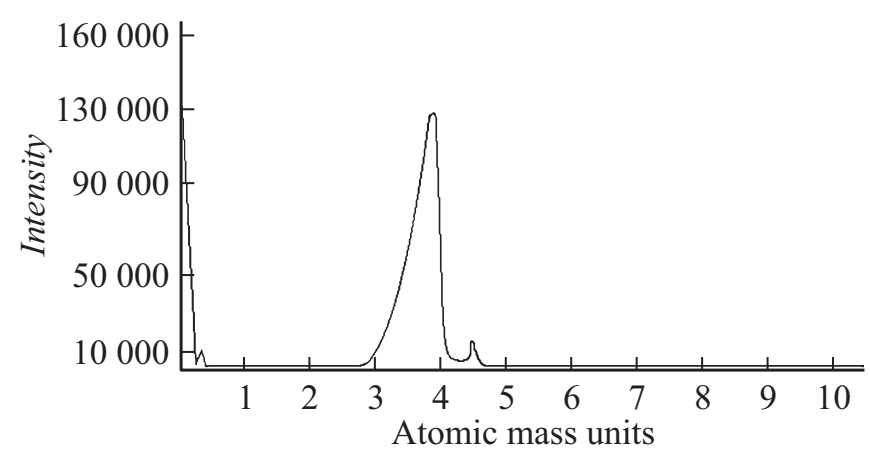

Рис. 1. График формирования пиков масс-спектрометра при дефекте ВДК. 
В процессе пайки и отжига в технологической среде водорода происходит обезгаживание металлов и очистка от поверхностных окислов. Режиму непосредственно самой пайки оболочки предшествовали два режима выдержки приборов в атмосфере водорода (при температуpe $700^{\circ} \mathrm{C}$ в течение $10 \mathrm{~min}$ и при температуре $750^{\circ} \mathrm{C}$ в течение $15 \mathrm{~min})$.

После выдержки приборов в водородной печи при температуре $810^{\circ} \mathrm{C}$ продолжительностью $5 \mathrm{~min}$ изделие нагревалось до температуры порядка $815^{\circ} \mathrm{C}$ (температуры плавления припоя). После того как металлокерамические узлы оболочки дугогасительной камеры соединялись, устанавливался режим, направленный на постепенное охлаждение изделия.

Из-за различия теплофизических свойств фаз в системе сплава $\mathrm{Cu}-\mathrm{Cr}$ и их температурных изменений при фазовых трансформациях, сопровождающих плавление и испарение, нагрев зоны катодного пятна дугового разряда рассчитать затруднительно. Поэтому экспериментально исследовались изменения морфологии (методом $\mathrm{ACM}$ ) и состава поверхности электродов (метод РСМА) после воздействия на них вакуумного дугового разряда. Изменением величины ускоряющего напряжения электронов зонда, изменяющего глубину его проникновения (при анализе методом РСМА глубина сбора информации о составе составляла $2 \mu \mathrm{m}$ при напряжении $1.30 \mathrm{kV}$ ), скрайбированием участков поверхности выявлялись изменения в атомных концентрациях основных и примесных составляющих контактов как по глубине, так и по поверхности. В частности, методом РСМА изучалось перераспределение атомных компонент в зоне взрывного расплавления электродов. Полученные результаты атомного состава электродов усреднялись программно для каждого спектра сканирования, и представлены в работе в виде усредненных значений.

\section{2. Результаты и их обсуждение}

Развитие дуговой стадии разряда обусловлено полевой эмиссией в зоне и в окрестности поверхности эмиттера с большими факторами усиления поля в области прикатодного падения потенциала (электрическое поле в этой зоне концентрируется на малом межэлектродном пространстве дугового разряда, примыкающего к катоду). Поэтому даже при падении напряжения в дуге величиной порядка $10-20 \mathrm{~V}$, напряженность электрического поля у катодов достигает порядка $10^{5} \mathrm{~V} \cdot \mathrm{cm}^{-1}$ и более. Формирование дуги обеспечивается превышением пороговых значений токов и напряжений для данных условий вследствие ионизации и зависит от параметров цепи и условий, а гашение дуги переменного тока достигается деионизацией плазмы между электродами и нулевыми мгновенными значениями тока в цепи дугового разряда. Высокая плотность тока приводит к разогреву центров эмиссии электронов вплоть до их расплавления, что сопровождается ростом десорбции и ионизации атомов - сначала атомов и молекул поверхностных адсорбированных слоев, а затем материалов контактов с выходом атомов в межэлектродное пространство и значительным нарастанием термоэлектронной составляющей тока.

\section{1. Результаты РСМА-исследования}

Из полученных методом РСМА результатов следует, что в момент плавления происходит перераспределение атомов меди от центра (точки воздействия плазменной дуги) к периферийным зонам с более низкой температурой. Наиболее существенные изменения поверхности электрода наблюдаются в зонах взрывного оплавления. Эта область принимает на себя энергетические потоки за счет воздействия ионов, ускоренных в прикатодном пространстве, и инициирования дугового разряда автоэлектронами. В центрах термоэмиссии (выступах поверхности) формируются высокие плотности тока,

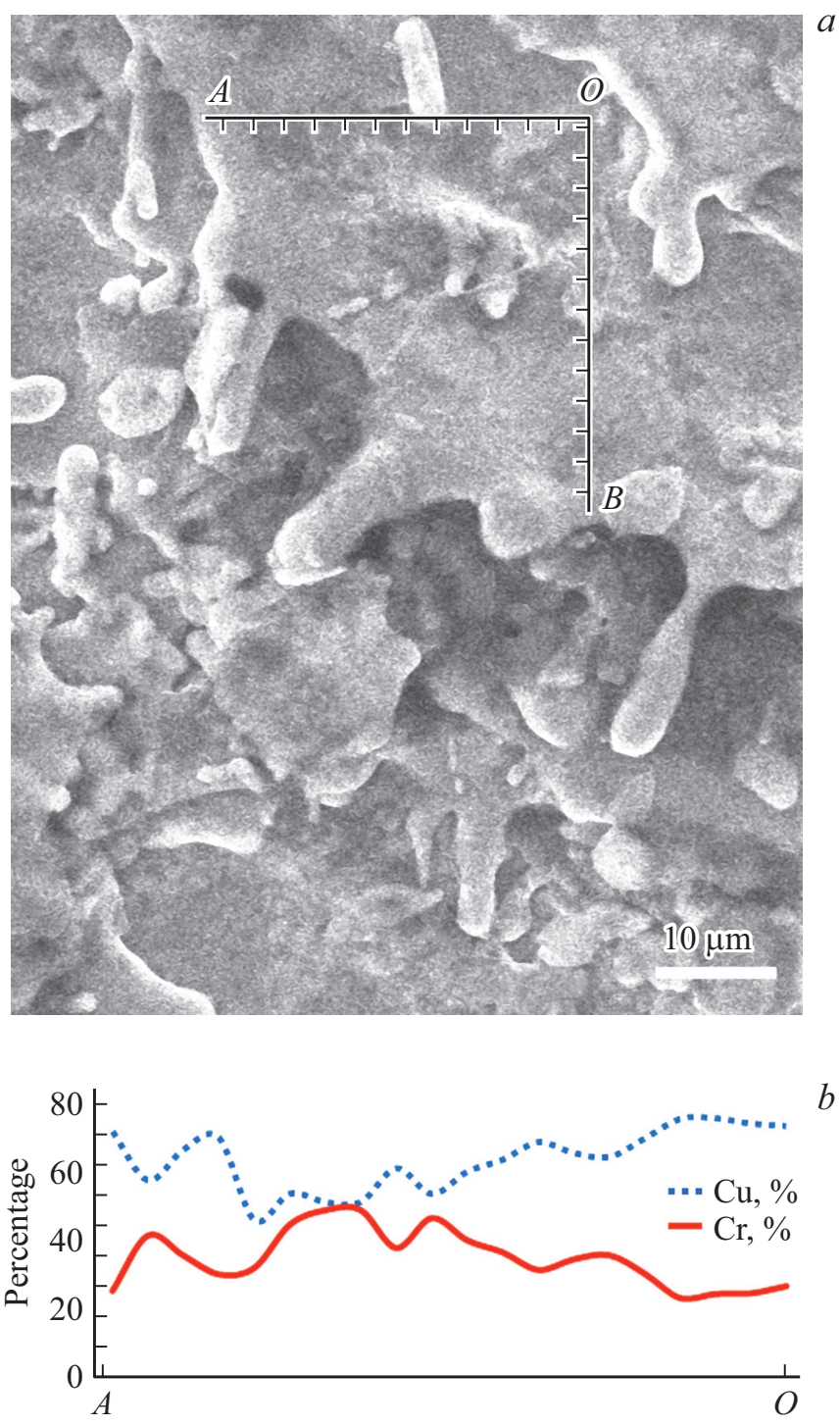

Рис. 2. Морфология оплавленной зоны $(a)$ и распределение ее атомного состава $(b)$. 
стимулирующие нагрев этой зоны. Энергия этих двух потоков, вызванных авто- и термоэлектронной эмиссиями, приводит к резистивному нагреву в зоне плавления. Этот процесс сопровождается резким увеличением объема, что приводит к разбрызгиванию поверхностной зоны. Отметим, что в области плавления (рис. 2) концентрация кислорода уменьшается более, чем в 2 раза (от 4.9 до 2.2 at.\%).

Этот результат, очевидно, связан с плавлением вещества в условиях вакуума, что приводит к сублимации окисных поверхностных соединений. Их распад происходит, когда скорость поступления тепловой энергии превышает скорость теплоотведения в поверхностном слое композиционного сплава. Медь обладает меньшим поверхностным натяжением и температурой плавления по сравнению с компонентой хрома в жидком состоянии, что позволяет атомам хрома, концентрируясь преимущественно в центре зоны плавления, выталкивать атомы меди на края этой зоны.

\section{2. Результаты исследования электронным зондированием}

Электронное зондирование образцов выявило зоны, содержание меди в которых снижается с 92.4 до 78.2 at.\% с увеличением глубины, что указывает на резко выраженный процесс ее сегрегации на поверхность. Это можно объяснить меньшей величиной поверхностного натяжения атомной компонентой меди по сравнению с хромом, что обеспечивает понижение энергии сплавной композиции в целом. Переход же в жидкое состояние приводит к процессу расслаивания сплава. Очевидно, что кинетически значительное увеличение коэффициента диффузии атомов меди в жидком виде (составляющего $10^{-5}-10^{-4} \mathrm{~cm}^{2} \cdot \mathrm{s}^{-1}$ ) способствует данному перераспределению основных составляющих сплава.

Рельеф, полученный при сканировании поверхности электродов вакуумной дуговой камеры в местах локального взрывного плавления, дополнялся растровым исследованием морфологии (в разных режимах ускоряющих напряжений) для анализа состава поверхности и объема образцов. Благодаря этому, можно было наблюдать результаты перераспределения атомов в зоне катодных пятен в электродах на основе системы $\mathrm{Cu}-\mathrm{Cr}$ с превышением атомной концентрации меди (71.8 вместо 53.7 at.\%) против начального ее значения. Очевиден факт сегрегации атомов материала электродов не только в системе объем-поверхность, но и в пятне взрывного расплавления электродов.

Кроме того, изучение морфологии поверхности и ее состава показывает на крайне неоднородный характер атомного состава верхнего слоя, особенно в местах расплавления электродов, что подтверждают также данные по составу основных и сопутствующих примесей. Установлено двукратное снижение концентрации кислорода в процессе деградации электрода (с 5.0 до 2.2 at.\%) и его уход из объема электродов. Кислород предпочтительно сконцентрирован в зонах с преобладанием легко окисляемых компонентов (в анализируемой системе это медь). Медь при протекании физико-химических реакций раньше и в большей степени стремится к окислению, что объясняет, в том числе, одну из причин выхода (сегрегацию) атомов $\mathrm{Cu}$ из объема электрода на поверхность.

\section{3. Результаты масс-спектрометрических исследований}

В настоящей работе авторы предлагают уточненную картину развития дуги с определением значимости роли поверхностных адсорбированных атомных слоев в процессе развития дугового вакуумного разряда. В частности, в работах Е.М. Окса с сотрудниками [14-17] регистрировались молекулы водорода в модификации $\mathrm{H}_{3}^{+}$ (в ходе развития разряда). Они наблюдались для особого состояния вакуумной дуги при увеличенных значениях тока и давления в разрядном промежутке. Нами же в ходе тестирования герметичности устройств в поверхностных слоях были обнаружены в рабочей среде молекулы остаточного гелия $\mathrm{He}^{+}$и молекул $\mathrm{H}_{3}^{+}$уже после проведения операций по проверке качества герметичности оболочки и водородного отжига деталей ВДК, а также после коммутационных испытаний. Хотя молекулы $\mathrm{H}_{3}^{+}$, вероятно, формируются предположительно на стадии водородного отжига деталей ВДК и регистрируются в дальнейших процедурах анализа остаточной атмосферы квадруполем, следует предположить, что они входят в состав поверхностных атомных слоев всех деталей вакуумного прерывателя. Поэтому из факта наблюдения в спектре квадрупольного масс-спектрометра линий $\mathrm{He}^{+}$ и $\mathrm{H}_{3}^{+}$следует, во-первых, вывод об эффекте наследования технологических примесей от предшествующих операций; во-вторых, можно высказать предположение,
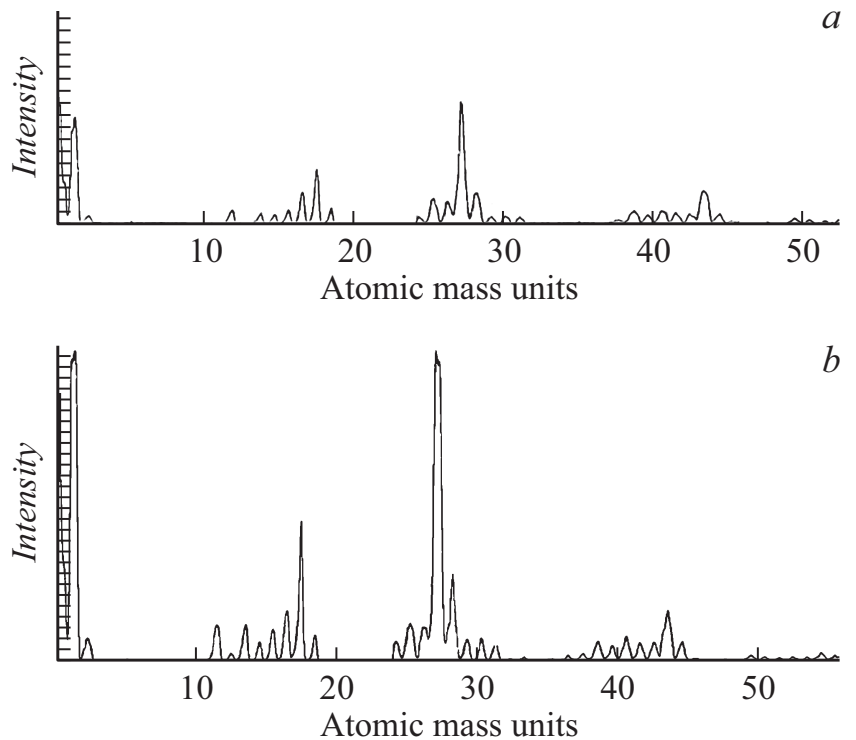

Рис. 3. Масс-спектр камеры до $(a)$ и после $(b)$ прогрева. 
что инициатором дугового разряда в вакуумном промежутке на начальной стадии, скорее всего, служат не только атомные составляющие материала электродов, но и компоненты поверхностных адсорбированных слоев, накопленные в ходе предшествующих технологических воздействий.

Помимо этого, в спектрах (рис. 3) найдены как ионные линии технологических газов, так и линии, соответствующие диффузионным маслам типа $\mathrm{C}_{x} \mathrm{H}_{y}^{+}$, молекулам, десорбируемым со всех деталей ВДК, и ионам остаточного газа $\left(\mathrm{CO}_{2}^{+}, \mathrm{H}_{2} \mathrm{O}^{+}, \mathrm{N}_{2}^{+}, \mathrm{N}^{+}\right.$и их полиатомных композиций). Большая массовая доля водорода объясняется применяемой технологией при производстве вакуумных камер, включающей, в частности, операции пайки и отжига в водородной печи.

Ожидаемым результатом явилось наблюдение изменения состава газа в процессе дегазации и пайки (разогрев до $815^{\circ} \mathrm{C}$ ). В процессе производства камеры линии остаточной атмосферы (водорода и воды) закономерно уменьшались, а линии оксидов углерода и азота увеличивались. При этом в установившемся режиме операций пайки ВДК все линии масс-спектра продолжают обнаруживать рост, что, вероятно, связано с интенсивным выделением газа уже из жидкой фазы с разрушением связей хемосорбированных состояний.

\section{Заключение}

Методом РСМА обнаружено доминирование атомов меди в характере перераспределения атомных компонент электродного материала между объемными и поверхностными слоями, а также в зоне взрывного расплавления электродов под действием вакуумного дугового разряда. Регистрация ионных составляющих масс-спектра квадрупольной системой в остаточной атмосфере вакуумной камеры на всех технологических этапах дает возможность обнаружить воздействие предшествующей обработки и по-новому интерпретировать роль адсорбированных слоев и электродных включений в развитии начальной стадии инициирования вакуумной дуги.

\section{Конфликт интересов}

Авторы заявляют, что у них нет конфликта интересов.

\section{Список литературы}

[1] Бучин В.А., Зериер М.П. // ЖТФ. 1990. Вып. 60. № 4. С. 92.

[2] Воронин А.В., Александров А.Е., Бер Б.Я., Брунков П.Н., Борматов А.А., Гусев В.К., Демина Е.В., Новохацкий А.Н., Павлов С.И., Прусакова М.Д., Сотникова Г.Ю., Яговкина М.А. // ЖТФ. 2016. Вып. 86. № 3. С. 51.

[3] Ерошкин М.В., Киселев Г.В., Моос Е.Н. // Изв. РАН. Сер. физ. 2014. Т. 78. № 6. С. 686.
[4] Борисовский П.А., Киселев Г.В., Киселева Л.И., Моос Е.Н., Наумкин А.В. // Поверхность. Рентгеновские, синхротронные и нейтронные исследования. 2017. № 5. С. 82.

[5] Benilov M.S., Marotta A. // J. Phys. D: Appl. Phys. 1995. Vol. 28. P. 1869.

[6] Benilov M.S. // IEEE Trans. Plasma Sci. 1994. Vol. 22. P. 73.

[7] Смоланов Н.А. // Поверхность. Рентгеновские, синхротронные и нейтронные исследования. 2018. № 6. С. 83.

[8] Духопельников Д.В., Воробьев Е.В., Ивахненко С.Г., Ахметжсанов Р.В., Обухов В.А., Попов Г.А., Хартов С.А. // Поверхность. Рентгеновские, синхротронные и нейтронные исследования. 2016. № 1. С. 15.

[9] Арустамов В.Н., Ашуров Х.Б., Миркаримов А.М., Пожаров С.Л., Кадыров Х.Х., Худайкулов И.Х. // Поверхность. Рентгеновские, синхротронные и нейтронные исследования. 2013. № 11. С. 95.

[10] Данилов M.E. Влияние материала контактов на отключающую способность дугогасительной камеры // Сборник статей сотрудников ВЭИ им. В.И. Ленина „Вакуумные дугогасительные камеры“. 2008. С. 68.

[11] Репин П.Б., Егоров Н.В. // ЖТФ. 2015. Т. 85. Вып. 2. С. 48.

[12] Селикатова С.М., Лукацкая И.А. Начальная стадия вакуумной дуги отключения // Сборник статей сотрудников ВЭИ им. В.И. Ленина „Вакуумные дугогасительные камеры“. 2008. С. 18.

[13] Kudyukin A.I., Moos E.N., Rott A.T. and others. Proceeding of 11th International Vacuum Electron Sources Conference // Arc Interaction with Electrodes. 2016. P. 41.

[14] Николаев А.Г., Окс Е.М., Фролова В.П., Юиков Г.Ю., Шмелев Д.Л., Уйманов И.В., Баренгольи, С.А. // ЖТФ. 2017. Т. 87. Вып. 5. С. 681.

[15] Казаков А.В., Медовник А.В., Бурдовищин В.А., Окс Е.М. // ЖТФ. 2015. Т. 85. № 2. С. 55.

[16] Николаев А.Г., Окс Е.М., Юшков Г.Я. // ЖТФ. 1998. Т. 68. № 5. C. 39.

[17] Визир А.В., Окс Е.М., Шандриков М.В., Юиков Г.Ю. // ЖТФ. 2017. Т. 87. Вып. 3. 